\title{
A Survey of Strategies, and Procedures for Detecting Drone Utilizing Sound, Radar, Thermal Infrared Sensor and Video Applications
}

\author{
Hassan Aldawsari \\ Electrical and Computer Engineering Department \\ King Abdulaziz University \\ Jeddah, Saudi Arabia \\ haldosari0012@stu.kau.edu.sa
}

\begin{abstract}
With the exponential rise in the use of drones anywhere anytime, malicious use by outlaws is increasing as well. This calls for protective, detective, preventive measures to counter these attacks. This paper aims to review the literature on drone detection and classification that utilizes a myriad of modalities ranging from using thermal infrared sensors to radar detections. In addition, there is a summary of a detailed discussion on drone classification along with recent progress and development in drone detection using machine learning, all of which are performed in an attempt to identify means to thwart such attacks. Furthermore, some future research directions, related to this new field of study, are discussed.
\end{abstract}

Keywords-Drone detection, machine learning, radar, video sensors.

\section{INTRODUCTION}

Unmanned aerial vehicles (UAVs), hereinafter called a drone, is small and remotely controlled and can be useful and of benefit to the society. Examples of their usefulness are to deliver automated external defibrillators [1], to fight fires more effectively [2] and for lawenforcement purposes. Additionally, the affordability and the ease of use make drones convenient for entertainment and amusement purposes [3].

Nevertheless, such drones can also be misused, regardless of whether this is intentional or not, thus, compromising or jeopardizing the safety and security of others. In worst-case scenarios, this can cause severe structural damage to an aircraft in the case of a collision with a consumer-sized drone in mid-air, even if they were navigating at low to moderate speed.

With the growing use of UAVs, there has been a notable rise in the number of drone-related incidents, such as that at the Gatwick Airport and the Saudi Arabia gas installation attacks.
As a result, demand for inexpensive drone detection systems that could be used across various nodes and applications, is increasing as well, in order to offset the risk of infrastructure trespassing, sabotage, and even the possible human endangerment. Studies conducted by researchers at Dayton University show that operating drones in the neighboring areas of an airport can negatively impact airplane fuselage. The reason drones pose such a great risk lies in their relatively small shape and surface area as well as the fact that they remain seemingly motionless as they soar above their target. This issue is even more exaggerated by the low frame processing rate of surveillance cameras, which renders frame- by-frame image detection and comparison rather difficult, if not impossible [4].

\section{DRONE DETECTION METHODS}

\section{A. Thermal infrared sensors}

The work described in [5], from 2017, does not use any form of machine learning; rather, the drone detection and classification is done by a person in real-time who observes the video stream output. The sensor used is a low-cost FLIR Lepton 80x60 pixels thermal camera and, with this connected to a Raspberry Pi, the authors are able to detect three different types of drones as far away as 100 meters.

One of the authors points out that it is the battery, not the motors that is the most significant source of heat radiation Sensors in the visible range. Combining a video camera with a detector based on a convolutional neural network (CNN) is a common strategy for detecting drones, as described in recent scientific papers.

In one of these papers [6], published in 2017, the researchers conduct an in-depth analysis of the performance of six different CNN-models for the drone detection task. The paper includes metrics for training time and performance in frames per second, as well as precession and recall curves. 
The authors reach the following conclusion after comparing the six different solutions: "considering speed and accuracy trade- offs, YOLOv2 might be the most appropriate detection model in our system."

As it turns out, when studying the subject of drone detection in images in the visible range, the YOLOv2architecture [7] is very common.

\section{B. Acoustic sensors}

Reference [8] investigates the use of acoustic sensors to detect drones. To extract features from audio signals, the Fast Fourier Transform (FFT) is used.

However, the MFCC (Mel Frequency Cepstrum Coefficients) appear to be the most commonly used feature extraction technique. The MFCC is "a non-linear mapping of the original frequency according to the auditory mechanism of the human ear.

It is the most used audio feature in current recognition tasks" [9]. When using a 120-element microphone array, the maximum acoustic detection range reported in the reviewed paper is $290 \mathrm{~m}$.

Also included is a DJI Phantom 2 drone. It is worth noting that the DJI Flame Wheel F450 is detected by the microphone array at a distance of 160 meters [10].

\section{Radar}

Because RADAR is the most commonly used technology for detecting flying objects, it is not farfetched to apply it to the detection of drones. However, doing this with a RADAR- system designed to detect aircraft is not as simple as it may appear.

This is because those systems frequently incorporate a built-in feature that works at reducing undesired echoes from small, slow, and low-flying objects, and as such fail to detect the drones in question.

The small Radar Cross Sections (RCS) of mediumsized consumer drones are well described in [11], and the results show that flying birds have similar RCS, which can lead to false detection of such targets when attempting to detect drones.

\section{DRONE CLASSIFICATION USING RADAR BASED ON MACHAINE LEARNING}

From reference [12], the objectives that researcher followed when applying ML to radar signals is:

- Drone detection: This is implemented when data is annotated using two labels: no-drone versus drone.
- Birds vs drones Classification: In which case, bird vs drones are the two used labels.

Drone Classification based on characterization:

Here, classification is implemented using a particular drone feature such as its payload or rotor blade count.

- Classification of different types of drones: this is implemented when the number of used labels matches the number of types of drones.

\section{A. Drone detection}

Baker and Jahangir practically demonstrated how, when it comes to detection using radar, the need for ML increases [13]. The researchers used a holographic, 3-D, high-end radar that has a $10 \mathrm{~kW}$ transmission power and a $32 \times 8$-receiver array.

Because of the low RCS, probability detection was almost Zero, especially when utilizing standard radar configuration. The researchers then lowered the threshold of amplitude, permitting detection at lower Doppler frequencies.

As a result, true positive drone detection was significantly improved. However, the heightened radar sensitivity resulted in more frequent false positives of such other targets as birds, air-borne debris, surfaces, and other clutter.

To overcome this drawback, the authors to select time-domain characteristics including maximum height, the radial velocity (Doppler), acceleration, and change in acceleration (jerk) use machine learning. The probability of drone prediction was improved, reaching 88\%, coupled with a reduced rate (0) of false positive classification by training the binary decision tree model [12].

\section{B. Birds vs drones classification}

The importance of discriminating drones from birds was emphasized by Trovik [14] since both possess diminished radar cross sections and are likely to create confusion during surveillance in comparison to noncooperative drones.

They maintained that plastic-rotor and gliding birds might be falsely detected as drones. They inculcate how the main characteristic of UAVs is their insignificant MDS (micro-Doppler signature) and reduced RCS modulation. 
Table 1 Comparison of Radar Methods Based on ML for Drone Tracking and Detection

\begin{tabular}{|c|c|c|c|c|c|c|}
\hline Radar System & Range & Classes & Data & Features & Classifier & Results \\
\hline $\begin{array}{l}\text { K-band and X- } \\
\text { band } \\
\text { CW radar }\end{array}$ & $1.2 \mathrm{~m}$ & $\begin{array}{l}3 \text { classes: } \\
\text { Quadcopter, } \\
\text { helicopter, hex- } \\
\text { copter }\end{array}$ & $\begin{array}{l}720 \text { samples each } \\
\text { radar/drone }\end{array}$ & $\begin{array}{l}\text { PCA based } \\
\text { features }\end{array}$ & SVM & $\begin{array}{l}\text { Classification } \\
\text { accuracy: up to } \\
94.7 \%\end{array}$ \\
\hline S-band CW & $3 \mathrm{~m}$ & $\begin{array}{l}4 \text { classes : } 3 \\
\text { drones and } 1 \\
\text { no-drone class }\end{array}$ & 280 images & $\begin{array}{l}\text { Spectral } \\
\text { correlation } \\
\text { function } \\
\text { (SCF) of } \\
\text { MDS } \\
\end{array}$ & $\begin{array}{l}\text { Deep Belief } \\
\text { network } \\
(\mathrm{DBN})\end{array}$ & $\begin{array}{l}\text { Classification } \\
\text { accuracy: above } \\
90 \% \\
\text { when } S N R \geq 0\end{array}$ \\
\hline $\begin{array}{l}\text { Ka-band CW } \\
\text { radar }\end{array}$ & $\begin{array}{l}2 \mathrm{~m} \\
\text { inlab }\end{array}$ & $\begin{array}{l}2 \text { classes: } \\
\text { drone and } \\
\text { simulated bird } \\
\text { data }\end{array}$ & $\begin{array}{l}30-10 \text { second } \\
\text { trials } \\
\text { per drone, } \\
\text { simulatedbird } \\
\text { MDS }\end{array}$ & $\begin{array}{l}\text { Mean } \\
\text { spectrogra } \\
\text { m,SVD, } \\
\text { CVD }\end{array}$ & SVM & $\begin{array}{l}\text { Classificatio } \\
\text { n accuracy: } \\
96 \% \text { to } \\
100 \%\end{array}$ \\
\hline $\begin{array}{l}\text { X-band CW } \\
\text { radar }\end{array}$ & $\leq 30 \mathrm{~m}$ & $\begin{array}{l}11 \text { classes all } \\
\text { classesfor } \\
\text { drones }\end{array}$ & $\begin{array}{l}30 \text { seconds } \\
\text { recording for } \\
\text { eachdrone }\end{array}$ & $\begin{array}{l}\text { Eigenvector } \\
\text { and } \\
\text { Eigenvalue } \\
\text { of MDS }\end{array}$ & $\begin{array}{l}\text { Naïve Bayes, } \\
\text { linear and } \\
\text { non-linear } \\
\text { SVM }\end{array}$ & $\begin{array}{l}\text { Classificati } \\
\text { on } \\
\text { accuracy: } \\
\approx 95 \%\end{array}$ \\
\hline S-band BirdRad & $\begin{array}{l}0.3-0.4 \\
\mathrm{~km}\end{array}$ & $\begin{array}{l}4 \text { classes: two } \\
\text { drones and two } \\
\text { birds (3-D solo } \\
\text { and DJI } \\
\text { Phantom } \\
\text { II) }\end{array}$ & $\begin{array}{l}8000 \text { trail } \\
\text { samples }\end{array}$ & $\begin{array}{l}9 \\
\text { polari } \\
\text { metric } \\
\text { feature } \\
\mathrm{s}\end{array}$ & $\begin{array}{l}\text { Nearest } \\
\text { neighbor } \\
\text { classifier }\end{array}$ & $\begin{array}{l}\text { Classificati } \\
\text { on accuracy } \\
: 100 \%\end{array}$ \\
\hline $\begin{array}{l}\text { L-band } \\
\text { holographicradar }\end{array}$ & $\begin{array}{l}1 \mathrm{~km} \\
- \\
500 \\
\mathrm{~m}\end{array}$ & $\begin{array}{l}2 \text { classes: } 1 \text { drone } \\
\text { and } \\
1 \text { non- drone }\end{array}$ & 5-min flight & $\begin{array}{l}\text { Height, } \\
\text { max, height } \\
\text { acceleration } \\
\text {, jerk }\end{array}$ & Decision tree & $\begin{array}{l}\text { Detection } \\
\text { probability: } 88 \%\end{array}$ \\
\hline
\end{tabular}

As a result, they propose for drone detection making use of polarimetric features as described in meteorology and radar ornithology.

In an attempt to acquire sufficient experience and enhance generalizability, the authors apply a total of nine polarimetric parameters, which include differential and linear depolarization ratios, as well as co-polarization ratios.

Yet, reference datasets that cover the various modalities and that are widely recognized are still scarce in the field of drone classification.

They contend that researchers have, in most cases, if not all of them, used lab experiments, simulations, and even outdoor measurements to generate their own data.
They conclude that, to emulate authentic-like environments, lab-generated data such as acoustic data have to be combined with noise [14].

\section{Drone vs. drone Classification}

Features from MDS were the focus of Molchanov's et al. study, who extracted those features from the Eigenvectors and Eigenvalues.

To this end, a linear as well as an SVM of non- linear nature and the classifier Naive Bayes have been developed.

They collected data by using a CW radar and they flew items of the following characteristics for half an each minute, with 11 items as follows: 
a) One artificial bird

b) One Quad Rotor

c) Two Fixed Wings

d) Three Helicopters

e) Four Stationary Rotors

Using 10-fold cross-classification, drone classification was accurate, on average, $95 \%$ of the time. Then a few models were excluded from, in a subsequent test, from being trained.

Yet, the authors discovered that classification was successful with the helicopter, stationary rotor, and fixedwing with $87 \%-100 \%$ accuracy [15].

The classification of Inspire 1 and F820 drones were investigated in [8] using a CNN (convolutional neural network) that has been pre-trained.

While hovering above an FMCW radar that had a KuBand of varying heights, ranging from 50 to one hundred meters, they calculated the CVD and recorded the MDS. Both images were merged into one image which was then renamed the MDI (Doppler image).

Ten thousand outdoor measurements were used to generate images. Then they were fed into the CNN classifier while applying a 4-fold cross validation. Surprisingly, results demonstrate that the drone classification accuracy reached 100\% [12].

\section{Drones characteristics Classification}

To determine whether the payload of a drone was one of three classes (0, 200, or 500 grams), Fioranelli et al. used machine learning to make this determination. They claimed that knowing a drone's payload can be used as an indication of malicious or hostile activity by suspicious users.

The data of a drone hovering for half a minute at a distance of 60 meters above a transceiver was recorded using 3 different recorders. As a result, each receiver yielded 15 samples for each payload class.

Both discriminant analysis and Naive Bayes were implemented, while maintaining training and testing cross validation. The three decision strategy results were displayed as follows:

i. The first model was obtained from receiver of the data that was collocated near the transmitter (i.e. the mono-static receiver)

ii. The model obtained after merging data recorded by the three receivers. iii. Three receivers yielded one model, each that was chosen after casting votes, and selecting the model with the highest voting. This resulted in $90 \%$ to $100 \%$ accuracy.

With respect to the tested classifiers and in terms of accuracy, Naïve Bayes was outperformed by discriminant analysis.

This was also observed with the increasing payload, where the authors noted that the MDS seemed to be "more uniform and straighter" reaching higher values, both positive and negative which attributed to the good classification results.

They explain this further by stating that more blade speed is required for higher payload to maintain the same altitude.

The authors conducted a subsequent study where they extracted MDS's centroid and the SVD. Moreover, they inserted a random forest to the current set of classification tools [16].

They found that MDS is able to generate synthetic data "for one to four propeller drones with 2-3 blades each".

An ANN- based MLP classifier was then trained using this data; this in turn was used to estimate the rotation rate and blade length through using regression.

SNR with varying levels were used to simulate authentic background noise. Classification was very accurate with SNRD5 at 99\%, whereas minimal errors were noted regarding parameter estimation with $4 \%$ for frequency of rotation and $6 \%$ blade length estimation ability [12].

\section{LIMITATIONS AND ADVANTAGES OF VARIOUS DRONE DETECTION TECHNOLOGIES}

There are many ways to detect drones, but it is difficult to say that one of these methods is completely better than the other.

Each method has a feature that differs from the other, as well as the choice of methods depends on the resources you have and the available tools.

In table 2, a comparison of some types depend on advantages and limitation are shown. 
Table 2 Comparison of Advantages and Limitations of Detection technology

\begin{tabular}{|c|c|c|c|}
\hline \multicolumn{4}{|c|}{ Detection Technology } \\
\hline & Radar & Visual & Acoustic \\
\hline Advantages & $\begin{array}{l}\text { - Low cost (FMCW) / Low } \\
\text { range. } \\
\text { - Radar resistant to fog, } \\
\text { dust, andcloud. } \\
\text { - It can work without need } \\
\text { to line-of-sight (LOS). } \\
\text { - Simultaneous detection } \\
\text { and trackingof multiple } \\
\text { drones }\end{array}$ & $\begin{array}{l}\text { - Depending on the used } \\
\text { camera and optical sensors } \\
\text { ithas low-cost. } \\
\text { - It easy for human } \\
\text { assessment of detection } \\
\text { results using screens. } \\
\text { - Can record visual evidence }\end{array}$ & $\begin{array}{l}\text { - It can work without need to line- } \\
\text { of-sight (LOS). } \\
\text { - Low-cost. }\end{array}$ \\
\hline Limitations & $\begin{array}{l}\text { - UAVs have small RCSs } \\
\text { that makedetection more } \\
\text { demanding. } \\
\text { - Some types of it are } \\
\text { expensive. } \\
\text { - Differentiation between } \\
\text { birds anddrones more } \\
\text { difficult. }\end{array}$ & $\begin{array}{l}\text { - It requires LOS } \\
\text { - The controllers cannot be } \\
\text { detectable. } \\
\text { - Affected by the weather }\end{array}$ & $\begin{array}{l}\text { - Needs a large dataset. } \\
\text { - Short operation range. } \\
\text { - Affected by the environment. }\end{array}$ \\
\hline
\end{tabular}

\section{CONCLUSION}

With the growth of commercial drones commercially that include such key applications as, agriculture, infrastructure and transport, media, telecommunication, to name a few, drone operation is posing great threats and is becoming a pending hazard to both assets and people. Moreover, while experts are exerting tremendous efforts on enforcing strict rules to ensure people and asset safety, malicious users are hard to control and thus more disruptions are recorded. Particularly, in low-altitude fields, a growing need for systems capable of keeping an overview of incidences, of running ongoing risk assessments, and even intercepting any attempts of infringement; the major goal being able to detect drones while roaming the skies and being able to classify them too. This becomes a very challenging feat with the soaring numbers of drones in the skies. As demonstrated in this review, it is expected that machine learning, as an integral part of $\mathrm{AI}$, will have a pivotal role in overcoming this. The different digital processing modalities is making ML an integral part of detection systems given that system operators pay close attention to data. The issue of drone detection, and more importantly its classification, continues to be labelled as urgent due to the high business demand and the high risk they pose to neighboring airborne objects, including planes and even wild life. Ongoing synergetic efforts are much needed to construct robust classification models that can be applied to all modalities. While no single modality has been identified as ideal for drone classification and detection, bi- modal and multi-modal systems have been suggested as they have shown promising results. The risk posed by drone operation is heavily linked to the drone's location and its proximity to critical areas. Therefore, range and altitude should be a priority. Focus, as has been demonstrated in this review, is allotted to detection predictability. There have been no studies that investigated drone classification applying distance as a classifying function using regression models, which can be a very interesting future research focus.

\section{REFERENCES}

[1] J. Sanfridsson et al. Drone delivery of an automated external defibrillator - a mixed method simulation study of bystander experience. Scandinavian Journal of Trauma, Resuscitation and Emer- gency Medicine, 27, 2019. Avalable : https://doi.org/10.1186/s13049-019-0622-6

[2] M. Innocente and P. Grasso. Self-organising swarms of firefighting drones: Harnessing the power of collective intelligence in decentralised multi-robot systems. Journal of Computational Science, 34:80-101, 2019.Avalable: https://www.sciencedirect.com/science/article/abs/pii/S1877750 318310238 ?via\%3Dihub

[3] The World Air Sports Federation FAI. World drone racing championship.Avalable: https://www.fai.org/world-cups/ droneracing-2020,

[4] Aviation International News. What happens when a drone hits an airplane wing? About the University of Dayton research institute. https://www.youtube.com/watch?v=QH0V7kp-xg0,

[5] P. Andrasi et al. Night-time detection of UAVs using thermal infrared camera. Transportation Research Procedia, 
28:183-190, 2017. Available: https://www.researchgate.net/publication/322914178_Nighttime _Detection_of_UAVs_using_Thermal_Infrared_Camera

[6] J. Park et al. A comparison of convolutional object detectorsfor real-time drone tracking using a PTZ camera. 17th Interna- tional Conference on Control, Automation and Systems (ICCAS 2017),pages 696-699, 2017.Avalable: https://www.semanticscholar.org/paper/A- comparison-ofconvolutional-object-detectors-for-Park-

Kim/f6012eab1f843cab564823aee8d0cf3ad4a82d69

[7] J. Redmon and A. Farhandi. YOLO9000: Better, faster, stronger. IEEE Conference on Computer Vision and Pattern Recognition (CVPR), 2017.Avalable https://ieeexplore.ieee.org/document/8100173

[8] J. Kim et al. Real-time UAV sound detection and analysis system.IEEE Sensors Applications Symposium (SAS), 2017.Avalable: https://www.researchgate.net/publication/316354424_Realtime_UAV_sound_detection_and_analysis_system

[9] H. Liu et al. Drone detection based on an audio-assisted cameraarray. IEEE Third International Conference on Multimedia Big Data (BigMM), 2017.Avalable : https://ieeexplore.ieee.org/document/7966780

[10] J. Busset et al. Detection and tracking of drones using advanced acoustic cameras. Proc. SPIE 9647, Unmanned/Unattended Sensors and Sensor Networks XI; and Advanced Free-Space Optical Communication Techniques and Applications, $2015 . \quad$ Available https://www.dora.lib4ri.ch/empa/islandora/object/empa\%3A117 62/da tastream/PDF/Busset-2015-

Detection_and_tracking_of_drones$\% 28$ published_version $\% 29$. pdf

[11] J. Gong et al. Interference of radar detection of drones by birds.Progress In Electromagnetics Research M, 81:1-11, 2019.Avalable https://www.jpier.org/PIERM/pier.php?paper=19020505

[12] B. Taha, A. Shoufan: ML-Based Drone Detection and Classification: State-of-the-Art in Research IEEE Access2019.Avalable https://www.semanticscholar.org/paper/Machine-LearningBased- Drone-Detection-and-in-TahaShoufan/69e21a7c05e72062b95e828c156d31a0d2b40712

[13] M. Jahangir and C. Baker, "Robust detection of micro-UAS drones with L-band 3-D holographic radar," in Proc. IEEE Sensor Signal Process. Defence (SSPD), Sep. 2016, pp. 1_5.Avalable: https://www.semanticscholar.org/paper/RobustDetection-of-Micro- UAS-Drones-with-L-Band-JahangirBaker/ae2f16ea5c3f270908d5d1f9128641bfb39215f8

[14] B. Torvik, K. E. Olsen, and H. Grif_ths, "Classification of birds and UAVs based on radar polarimetry," IEEE Geosci. Remote Sens. Lett., vol. 13,no. 9, pp. 1305_1309, Sep. 2016. Avalable : https://ieeexplore.ieee.org/document/7508914

[15] P. Molchanov, R. I. A. Harmanny, J. J. M. de Wit, K. Egiazarian, and J. Astola, "Classi_cation of small UAVs and birds by microDoppler signatures," Int. J. Microw. Wireless Technol., vol. 6, nos. 3_4, pp. 435_444,Jun. 2014.Avalable https://www.cambridge.org/core/journals/international-journalof- microwave-and-wirelesstechnologies/article/abs/classification-of- small-uavs-and-birdsby-microdopplersignatures/C5A57FC02BBC4261CE563738ED9D6D76

[16] M. Ritchie, F. Fioranelli, H. Borrion, and H. Grif_ths, "Multistatic micro-Doppler radar feature extraction for classi_cation of unloaded/loadedmicro-drones," IET Radar, Sonar Navigat., vol. 11, no. 1, pp. 116_124,Jan. 2017.Avalable : https://doi.org/10.1049/iet- rsn.2016.0063 